\title{
NERVE ENDINGS AND VASCULAR SUPPLY IN SEMITENDINOSUS TENDON OF CEREBRAL PALSY CHILDREN
}

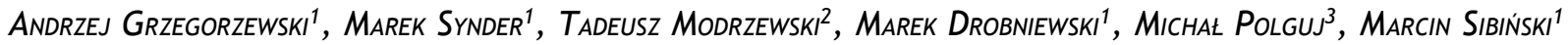

\section{ABSTRACT}

Objective: To evaluate the distribution of SP (substance P) and S-100 peptide immunoreactivity, as well as the vascular supply of tissues commonly used as grafts for anterior cruciate ligament $(\mathrm{ACL})$ reconstruction. A second aim was to compare the above mentioned distribution in the semitendinosus muscle tendons of cerebral palsy (CP) patients with the semitendinosus muscle tendons and patellar tendons of patients without CP. Methods: The first group consisted of 14 children with cerebral palsy with a mean age of 11.7 years old. At the time of hamstring lengthening operation, a sample of semitendinosus muscle was taken for analysis. The second group comprised 20 patients treated for isolated ACL rupture of the knee (mean age 32 years old). Group three comprised ten patients in the mean age of 14.3 years old treated for recurrent lateral patellar dislocation, and from whom a sample of patellar tendon was obtained. Results: No statistically significant differences were demonstrated with regard to the amount of immunopositive nerve fibers expressing SP or S-100 in all 3 groups of patients. A significant difference was noted in the number of blood vessels between the adult and child semitendinosus muscles, but not between the semitendinosus muscles and patellar tendon of children. Conclusion: The number of nociceptors as well as proprioceptive fibers is similar in patients with $\mathrm{CP}$ and patients from a neurologically healthy population. Level of Evidence IV, Cases Series.

Keywords: Cerebral palsy. Patellar dislocation. Anterior cruciate ligament. Rupture.

Citation: Grzegorzewski A, Synder M, Modrzewski T, Drobniewski M, Polguj M, Sibiński M. Nerve endings and vascular supply in semitendinosus tendon of cerebral palsy children. Acta Ortop Bras. [online]. 2015;23(5):259-62. Available from URL: http://www.scielo.br/aob.

\section{INTRODUCTION}

Anterior cruciate ligament (ACL) reconstruction has been performed in a broad range of patients in recent years. This operation is now a standard procedure with favourable results regardless of age, gender or occupation, thanks to advances in surgical techniques, instrumentation and basic research. Tajima et al. reported a case of ACL reconstruction in a 25-year-old patient with cerebral palsy (CP) using an autologous hamstring double-bundle technique. Preoperative symptoms of instability were resolved and a high degree of satisfaction was achieved. ${ }^{1}$ These results raise the question on the suitability of tendon graft material from $\mathrm{CP}$ patients for reconstruction. In these subjects, the mechanical properties, vascularity and innervation of the graft may differ from the healthy population. Numerous previous studies have provided a qualitative and quantitative analysis of mechanoreceptors in the hip joint, or in knees with anterior knee pain syndrome or ACL rupture. ${ }^{2-10}$ However, knowledge on the distribution of nociceptors in tissues used for ACL reconstruction is still rather scarce.
The role of substance $\mathrm{P}$ (SP) as a neurotransmitter (particularly for free nerve endings) in the innervation of nociceptors of the soft tissue of the knee has been highlighted by many studies. ${ }^{5-7,11}$ Their relationship with anterior knee pain was first noted by Witoński et al., ${ }^{9}$ who reported a significantly greater number of these fibres in Hoffa pad as well as in the medial patellar retinaculum.

The S-100 protein family plays a major role in the development of nerve tissue, its functioning and the pathophysiology of nerve conduction disorders. ${ }^{12}$ S-100 proteins are a group of intracellular and extracellular transmitters, modulated by $\mathrm{Ca}^{2+}$ ions. They are a good marker of nervous structures in soft tissues of the knee joint, and were chosen for evaluation of nociceptors. ${ }^{12-14}$ The aim of the study was to evaluate the distribution of SP and S-100 peptide immunoreactivity as well as the vascular supply of tissues commonly used as grafts for $\mathrm{ACL}$ reconstruction. A second aim was to compare the above mentioned distribution in the semitendinosus muscle tendons of CP patients with

All the authors declare that there is no potential conflict of interest referring to this article.

\footnotetext{
1. Medical University of Lodz, Orthopedics and Pediatric Orthopedics Clinic, Lodz, Poland.

2. Medical University of Lodz, Pathomorphology and Clinical Cytopathology Department, Lodz, Poland.

3. Medical University of Lodz, Department of Angiology, Lodz, Poland.
}

This study was performed at the Medical University of Lodz, Orthopedics and Pediatric Orthopedics Clinic, Lodz, Poland.

Correspondence: Marcin Sibiński, Medical University of Lodz, OrthopedicS and Pediatric Orthopedics Clinic. Drewnowska, 75. 91-002, Lodz, Poland. sibinek@poczta.onet.pl

Article received in 11/14/2014, approved in 02/10/2015.

Acta Ortop Bras. 2015;23(5):259-62 
the semitendinosus muscle tendons and patellar tendons of patients without CP. We made the hypothesis that hamstring tendons of children with $\mathrm{CP}$ may have different nociceptive and proprioceptive properties or vascular supply comparing to a neurologically healthy population, what would make them unsuitable as a graft for ACL reconstruction. Better initial vascular supply and innervation could positively influence graft integration and future proprioception and vice versa, worse vascularity and low concentration of nerve endings would have negative effect on these two factors. To our knowledge, this is the first article to study this relationship.

\section{MATERIALS AND METHODS}

Tissue sample collection was performed in three groups of patients operated in our hospital in the years 2006-2008. Group one comprised ten patients (mean age 14.3 years, range: 1017 years) treated for recurrent lateral patellar dislocation, and from whom a sample of patellar tendon was obtained. All those patients ( 8 girls and 2 boys) had experienced a first traumatic dislocation followed by 4 to 6 small traumatic incidents. Conservative treatment was firstly introduced for the above mentioned dislocations: stabilizing orthosis, ice compresses, joint relief, analgesics and exercises. The final incident of patellar dislocation took place 6 months before surgery. Congenital knee joint abnormalities were excluded from the study. Open reconstruction of medial patellar stabilizers was performed by the Blauth procedure. ${ }^{15}$ Intraoperative defects in the knee joint were also excluded.

The second group consisted of 14 children ( 3 girls and 11 boys) with $\mathrm{CP}$ (mean age 11.7 years, range: 7-18 years). All these patients were treated for knee flexion contracture related to medial and lateral hamstring spasticity. During operation biceps femoris and semimebranosis lengthening were performed, and the semitendinosus muscle released. At the time of operation, a sample of semitendinosus muscle was collected for analysis. The third group comprised 20 patients (4 female and 16 male) treated for isolated ACL rupture of the knee (mean age 32 years; range 23-43 years). None of them had previously undergone operations in the knee joint region or had been administered intra-articular steroid injections. The patients reported knee joint instability, but no anterior knee pain nor symptoms related to other knee lesions. X-ray examination of the knee joint did not reveal any bony abnormalities. ACL reconstruction was performed arthroscopically by a double tunnel technique using medial hamstrings as graft. During the operation, semitendinosus and gracilis muscle tendons were harvested by antero-medial incision and a semitendinosus tendon sample was collected for analysis. Arthroscopic evaluation excluded anything other than ACL rupture knee abnormalities.

Tissue specimens, fixed in $4 \%$ formalin, were passed through a number of reagents in a Tissue-Tek VIP tissue processor (Sakura) for dehydration, fluoroscopic examination and paraffin saturation.

Such processed specimens were embedded into paraffin blocks by a histoembedder (Leica) and cut on a sliding microtome (Micron) into $4 \mu \mathrm{m}$ sections, which were then placed on silanised microscope slides. The slices were stained with haematoxylin and eosin $(H+E)$ in an automatic Autocolor I stainer (Micron) to be completed by immunohistochemical reactions with SP and S-100. H + E staining was performed as earlier described. ${ }^{16}$ The preparations were closed with cover glasses using the DPX Mounting Media \& Section Adhesive media.

Microscopic evaluation was done by the third author of the third study (TM), who was not involved in the process of operative treatment and material collection. An Olympus BX 50 light microscope (Olympus Corporation, Tokyo, Japan) was used. Approval for the study was obtained from the Commission of Bioethics of our University. Written informed consent for participation in the study was obtained from patients, the child's parents or guardians.

\section{Statistical analysis}

Statistical analyses were performed by means of SPSS 14.0 for Windows (SPSS Inc., Chicago, Illinois). The Fisher exact test was used to compare the composition of semitendinosus muscle between children with $\mathrm{CP}$ and adults regarding the distribution of immunopositive nerve fibers expressing S-100 or SP. The Fisher exact test was also used to compare the distribution of immunopositive nerve fibers expressing S-100 between samples excised from semitendinosus muscle in children with $\mathrm{CP}$ and the patellar ligament of children with patellar instability. The linear relationship test was used to compare the number of blood vessels between samples and the distribution of nerve fibers expressing SP in preparations collected from semitendinosus muscle in children with $\mathrm{CP}$ and the patellar ligament of children with patellar instability. Values of $p$ below 0.05 were regarded as statistically significant.

\section{RESULTS}

The mean values of nerve fibers expressing SP and S-100 protein, as well as the number of blood vessels, are presented in Table 1. A positive immunohistochemical reaction for SP fibers was found in four patellar tendons, 11 semitendinosus muscles from children with $\mathrm{CP}$ and 12 semitendinosus muscles from adults. A positive immunohistochemical reaction for S-100 fibers was obtained in four patellar tendons, seven semitendinosus muscles from children with $\mathrm{CP}$ and eight semitendinosus muscles from adults. Blood vessels were seen in all but one patellar tendon, all but one semitendinosus muscle from a child with $\mathrm{CP}$ and 11 out of 20 adult semitendinosus muscle. (Tables 2 and 3)

Table 1. Mean values from samples showing blood vessels, substance
$\mathrm{P}$ and S-100 protein expression in slices excised from the semitendi-
nosus muscle of children with cerebral palsy ( $\mathrm{n}=14)$, patellar ligament
of children with patellar instability $(\mathrm{n}=10)$ and semitendinosus muscle
in eight adults obtained during $\mathrm{ACL}$ reconstruction $(\mathrm{n}=20)$.


Table 2. Comparison of mean value from ten consecutive visual areas showing blood vessel numbers, Substance P and S-100 protein expression between samples excised from semitendinosus muscle in children with cerebral palsy $(n=14)$ and patellar ligament of children with patellar instability $(n=10)$.

\begin{tabular}{|c|c|c|c|c|}
\hline & Group & $\begin{array}{l}\text { Semitendinosus } \\
\text { muscle }\end{array}$ & $\begin{array}{c}\text { Patellar } \\
\text { ligament }\end{array}$ & $p$ value \\
\hline \multirow{2}{*}{ Substance P } & Negative & 3 & 6 & 0.196 \\
\hline & Positive & 11 & 4 & \\
\hline \multirow{2}{*}{ S-100 } & Negative & 7 & 6 & 0.473 \\
\hline & Positive & 7 & 4 & \\
\hline \multirow{4}{*}{$\begin{array}{l}\text { Mean number of } \\
\text { blood vessels }\end{array}$} & None & 1 & 1 & 0.174 \\
\hline & $\begin{array}{l}\text { Small } \\
\text { amount }\end{array}$ & 10 & 4 & \\
\hline & $\begin{array}{l}\text { Medium } \\
\text { amount }\end{array}$ & 3 & 4 & \\
\hline & Many & 0 & 1 & \\
\hline
\end{tabular}

Table 3. Comparison of mean value from ten consecutive visual areas showing blood vessels, Substance P and S-100 protein expression between samples excised from semitendinosus muscle in children with cerebral palsy $(n=14)$ and semitendinosus muscle in adults obtained during $A C L$ reconstruction $(n=20)$.

\begin{tabular}{c|c|c|c|c}
\hline \multirow{2}{*}{ Substance P } & Group & $\begin{array}{c}\text { Child } \\
\text { semitendinosus } \\
\text { muscle }\end{array}$ & $\begin{array}{c}\text { Adult } \\
\text { semitendinosus } \\
\text { muscle }\end{array}$ & p value \\
\cline { 2 - 5 } & Negative & 3 & 8 & 0.223 \\
\hline \multirow{3}{*}{ S-100 } & Positive & 11 & 12 & \\
\cline { 2 - 5 } & Negative & 7 & 12 & 0.4 \\
\cline { 2 - 5 } & Positive & 7 & 8 & \\
\cline { 2 - 5 } Mean number & None & 1 & 9 & $\mathbf{0 . 0 0 5}$ \\
\cline { 2 - 5 } of blood vessels & $\begin{array}{c}\text { Small } \\
\text { amount }\end{array}$ & 10 & 11 & \\
\cline { 2 - 5 } & $\begin{array}{c}\text { Medium } \\
\text { amount }\end{array}$ & 3 & 0 & \\
\hline
\end{tabular}

Values in bold are statistically significant.

No statistically significant differences were demonstrated with regard to the amount of immunopositive nerve fibers expressing SP or S-100 in the tissues collected from all three groups of patients. (Tables 2 and 3) A significant difference was noticed in the number of blood vessels between the adult and CP children semitendinosus muscles, but not between the semitendinosus muscles and patellar tendon of children. (Tables 2 and 3 )

\section{DISCUSSION}

$\mathrm{CP}$ is an irreversible distorter of the central nervous system which leads to a spectrum of dysfunctions and spasticity in most cases. Theoretically, it may be expected that muscles and tendons may have neural changes that will influence their mechanical properties, raising the question of whether these hamstring tendon grafts can be successfully used for ACL reconstruction.

Our analysis revealed no statistically significant differences between semitendinosus muscle in children with $\mathrm{CP}$ and the other two most popular grafts used for this procedure in healthy po-

Acta Ortop Bras. 2015;23(5):259-62 pulations: semitendinosus muscle tendon and patellar tendon. The only statistically significant factor was the better vascularity of semitendinosus tendon in patients with $\mathrm{CP}$ than those with ACL rupture. Our data shows that tendon grafts of $\mathrm{CP}$ children are as good as grafts of neurologically healthy people for $\mathrm{ACL}$ reconstruction. $\mathrm{ACL}$ injury of child with $\mathrm{CP}$ is a rare indication for reconstruction. However, indications for this procedure have been extended, and the population of $\mathrm{CP}$ patients may require it more often in the future. The good results of this procedure reported by Tajima et al. ${ }^{1}$ also confirm the fact that hamstring tendons can be safely and effectively used for this procedure. Unfortunately, it is difficult to compare our results to others', as no one performed similar to our investigations.

Using protein S-100 as a marker of nerve fibres, Masłoń et al. ${ }^{5}$ noticed that the distribution of nociceptor nerve fibres in the soft tissues of the knee joint, of which SP is the neurotransmitter, shrinks as the patient ages. Biedert et al. ${ }^{2}$ performed a histological comparison of 18 samples taken from 8 knee joints of cadavers. It was found, qualitatively and quantitatively, that most of the free nerve endings (type IV nerve fibres according to Freeman and Wake) were located in the lateral and medial patellar retinaculum, the patellar ligament, the pes anserinus, as well as the meniscofemoral ligaments (Wrisberg and Humphry). The number of free nerve endings positively correlated with the occurrence of clinical symptoms (incapacitating pain). Immunohistochemical analysis using SP as a marker revealed a statistically significant number of nerve fibres conducting pain in the Hoffa's bodies and the medial patellar retinaculum in patients with symptoms of anterior knee pain. ${ }^{5}$ Bohnsack et al. ${ }^{17}$ also report that Hoffa's bodies play a biochemical role in the course of anterior knee pain.,17

Witoński and Wągrowska-Danielewicz ${ }^{10}$ noted that distribution of the nociceptive nerve supply, positively stained for SP, is equally distributed throughout the intact $\mathrm{ACL}$. The density of nerve endings expressing SP was significantly affected by ACL injury as well as by the time since rupture. Dhillon et al. ${ }^{18}$ noticed that persistent residual proprioceptive fibers in injured ACLs, identified by monoclonal antibodies to S-100, are present. Thus, not shaving ACL remnants may be of potential benefit during $A C L$ reconstruction, as some re-innervation and recovery of proprioceptive potential may be possible, improving, thus, clinical outcomes. These findings suggest that a higher number of nociceptive nerve endings and proprioceptive fibers in tendons used as ACL grafts may have a positive influence on the outcome of $A C L$ reconstruction.

This paper has some weaknesses. It was not possible to collect samples from patients in the same age groups. Those who had ACL reconstruction, i.e. the semitendinosus muscle group, were older and those with recurrent patellar dislocation, i.e. the patellar tendon group, were younger than the study group. Furthermore, although the numbers of patients in each group is relatively low, they are sufficient for statistical analysis. Further research should be performed to compare the mechanical properties of the different grafts.

\section{CONCLUSION}

The number of nociceptors as well as proprioceptive fibers is similar in patients with $\mathrm{CP}$ and patients from a neurologically healthy population. 


\section{REFERENCES}

1. Tajima T, Chosa E, Yamamoto K, Kawahara K, Yamaguchi N, Watanabe S. Anterior cruciate ligament reconstruction in a patient with athetoid cerebral palsy: a case report. Sports Med Arthrosc Rehabil Ther Technol 2012;4(1):36 .

2. Biedert RM, Stauffer E, Friederich NF. Occurrence of free nerve endings in the soft tissue of the knee joint. A histologic investigation. Am J Sports Med.1992;20(4):430-3.

3. Bohnsack M, Hurschler C, Demirtas T, Rühmann O, Stukenborg-Colsman C, Wirth CJ. Infrapatellar fat pad pressure and volume changes of the anterior compartment during knee motion: possible clinical consequences to the anterior knee pain syndrome. Knee Surg Sports Traumatol Arthrosc. 2005;13(2):135-41.

4. Leunig M, Beck M, Stauffer E, Hertel R, Ganz R. Freenerve endings in the ligamentum capitis femoris. Acta Orthop Scand. 2000;71(5):452-4

5. Masłoń A, Witoński D, Modrzewski T, Grabicki M, Sibiński M, Grzegorzewski A. Phenomenon of painless knee in recurrent patellar dislocation in children. Arch Med Sci. 2014;10(3):531-6.

6. Sanchis-Alfonso V, Roselló-Sastre E, Monteagudo-Castro C, Esquerdo J. Quantitative analysis of nerve changes in the lateral retinaculum in patients with isolated symptomatic patella-femoral malalignment. A preliminary study. Am J Sports Med 1998;26(5):703-9.

7. Walsh DA, Salmon M, Mapp PI, Wharton J, Garrett N, Blake DR, et al. Microvascular substance $P$ binding to normal and inflamed rat and human synovium. J Pharmacol Exp Ther. 1993;267(2):951-60.

8. White DM, Helme RD. Release of substance $P$ from peripheral nerve terminals following electrical stimulationof the sciatic nerve. Brain Res .1985;336(1):27-31.

9. Witoński D, Wagrowska-Danielewicz M. Distribution of substance-P nerve fibers in the knee joint in patients with anterior knee pain syndrome. A preliminary report. Knee Surg Sports Traumatol Arthrosc. 1999;7(3):177-83.

10. Witoński D, Wagrowska-Danilewicz M. Distribution of substance-P nerve fibers in intact and ruptured human anterior cruciate ligament: a semi-quantitative immunohistochemical assessment. Knee Surg Sports Traumatol Arthrosc. 2004;12(5):497-502.

11. Lembeck F, Folkers K, Donnerer J. Analgesic effect of antagonists of substance P. Biochem Biophys Res Commun. 1981;103(4):1318-21.

12. Zimmer DB, Cornwall EH, Landar A, Song W. The S100 protein family: history, function, and expression. Brain Res Bull. 1995;37(4):417-29.

13. Fano G, Biocca S, Fulle S, Mariggio MA, Belia S, Calissano P. The S-100: a protein family in search of a function. Prog Neurobiol. 1995;46(1):71-82.

14. Kashani IR, Golipoor Z, Akbari M, Mahmoudi R, Azari S, Shirazi R, et al. Schwann-like cell differentiation from rat bone marrow stem cells. Arch Med Sci. $2011 ; 7(1): 45-52$

15. Blauth W, Mann M. Medial-and simultaneous anterior-transfer of the tibial tuberosity (author's transl). Z Orthop Ihre Grenzgeb. 1977;115(2):252-5.

16. Burck HC. Technika histologiczna. Warsaw: PZWL; 1975

17. Bohnsack M, Klages $P$, Hurschler C, Halcour A, Wilharm A, Ostermeier S, et al. Influence of an infrapatellar fat pad edema on patellofemoral biomechanics and knee kinematics: a possible relation to the anterior knee pain syndrome. Arch Orthop Trauma Surg. 2009;129(8):1025-30.

18. Dhillon MS, Bali K, Vasistha RK. Immunohistological evaluation of proprioceptive potential of the residual stump of injured anterior cruciate ligaments (ACL). Int Orthop. 2010;34(5):737-41. 